\title{
LETTER
}

\section{Cardiac arrest and chewing gum - an unfortunate combination}

Angus G Thompson, Shakeeb Razak and Rohan Jayasinghe

To THE EDITOR: We report a case of successful resuscitation after cardiac arrest associated with obstruction of the airway by chewing gum.

In December 2005, in Port Hedland, Western Australia, a 57-year-old electrician was found unconscious by workmates 5 minutes after he was seen working normally. He was not breathing and had no pulse. Bystander cardiopulmonary resuscitation (CPR) was commenced immediately and an ambulance arrived within 3 minutes. Cardiac monitoring showed the patient was in ventricular fibrillation. Direct-current defibrillation was performed three times, resulting in reversion to ventricular tachycardia, rapid atrial fibrillation and, within minutes, spontaneous reversion to sinus rhythm.

The time from ambulance dispatch to arrival at Port Hedland Regional Hospital was less than 15 minutes. Rapid transport to hospital enabled prompt assessment of the patient, who was found to be maintaining sinus rhythm with left bundle branch block but no ST segment changes. Following the return of spontaneous circulation and consciousness, the patient's
Glasgow Coma Score and oxygen saturation unexpectedly deteriorated. On rapid sequence intubation, chewing gum was found lodged in the patient's larynx; it was removed, and intubation completed. An urgent computed tomography scan found no intracranial cause for the deterioration in his condition. The patient was transferred to a tertiary centre by the Royal Flying Doctor Service.

There was a rise in the patient's troponin level after the cardiac event, but normal coronary arteries with globally depressed ventricular function were seen on angiography, and an ejection fraction of $30 \%$ on echocardiography, suggesting an underlying cardiomyopathy. An implantable cardiac defibrillator was inserted. The patient recovered, with a diagnosis of hypoxia associated with myopathic cardiac arrest complicated by laryngeal obstruction from chewing gum. He suffered a moderate hypoxic brain injury during the event and underwent a short period of inpatient rehabilitation, before returning to work within 6 months. He had no significant sequelae 18 months after the cardiac event.

There have been previous reports of sudden death due to airway obstruction by chewing gum in children and one recent report of adult death, but no reports of successful resuscitation after cardiac arrest and hypoxia associated with chewing gum obstruction. ${ }^{1,2}$ There have also been reports of delayed problems with ventilation due to migration of previously aspirated chewing gum. ${ }^{3}$ While it is unlikely that the chewing gum airway obstruction was the primary event in this case, given the finding of cardiomyopathy, we believe that the laryngeal obstruction contributed to the patient's secondary deterioration.

This case demonstrates the importance of CPR training in the community, rapid paramedical and medical response, and the need to consider complicating causes in the event of cardiac arrest when return of spontaneous circulation does not result in clinical improvement as expected. It is made all the more remarkable by its occurrence in a remote regional centre of northwest WA.

Angus G Thompson, Medical Registrar ${ }^{1}$ Shakeeb Razak, Electrophysiology Fellow ${ }^{2}$ Rohan Jayasinghe, Director of Cardiac Services and Cardiology ${ }^{3}$

1 Sir Charles Gairdner Hospital, Perth, WA.

2 Royal Perth Hospital, Perth, WA.

3 Gold Coast Hospital, Gold Coast, QLD. angus.thompson@health.wa.gov.au

1 Njau SN. Adult sudden death caused by aspiration of chewing gum. Forensic Sci Int 2004; 139: 103-106.

2 Steichen FM, Fellini A, Einhorn AH. Acute foreign body laryngo-tracheal obstruction: a cause for sudden and unexpected death in children. Pediatrics 1971; 48: 281-285.

3 Shlamovitz GZ, Halpern P. Delayed obstruction of endotracheal tubes by aspirated foreign bodies: report of two cases. Ann Emerg Med 2004; 43: 630-633. 